\title{
Androgen Receptor Mutations Are Associated with Altered Epigenomic Programming as Evidenced by HOXA5 Methylation
}

\author{
S. Bens ${ }^{a, b}$ \\ O. Ammerpohl ${ }^{a}$ J.I. Martin-Subero ${ }^{a}$ d \\ M. Appari ${ }^{b} \quad$ J. Richter ${ }^{a}$ \\ O. Hiort ${ }^{c}$ \\ R. Werner ${ }^{c}$ F.G. Riepe ${ }^{b}$ \\ R. Siebert ${ }^{a}$ \\ P.-M. Holterhus ${ }^{b}$ \\ ${ }^{a}$ Institute of Human Genetics and b Department of Pediatrics, Christian Albrechts University of Kiel and University \\ Hospital Schleswig-Holstein, Campus Kiel, Kiel, and ${ }^{\mathrm{C}}$ Department of Pediatric and Adolescent Medicine, University \\ of Lübeck and University Hospital Schleswig-Holstein, Campus Lübeck, Lübeck, Germany; ${ }^{d}$ Cancer Epigenetics \\ and Biology Program, Bellvitge Institute for Biomedical Research, Barcelona, Spain
}

\section{Key Words}

Androgen insensitivity syndrome $\cdot$ Androgen receptor mutation - DNA methylation - Epigenetic $\cdot$ HOXA5

\begin{abstract}
Male external genital differentiation is accompanied by implementation of a long-term, male-specific gene expression pattern indicating androgen programming in cultured genital fibroblasts. We hypothesized the existence of an epigenetic background contributing to this phenomenon. DNA methylation levels in 2 normal scrotal fibroblast strains from $46, X Y$ males compared to 2 labia majora fibroblast strains from 46, $X Y$ females with complete androgen insensitivity syndrome (AIS) due to androgen receptor (AR) mutations were analyzed by Illumina GoldenGate methylation arrays ${ }^{\circledR}$. Results were validated with pyrosequencing in labia majora fibroblast strains from fifteen $46, X Y$ patients and compared to nine normal male scrotal fibroblast strains. HOXA5 showed a significantly higher methylation level in complete AIS. This finding was confirmed by bisulfite pyrosequencing of 14 CpG positions within the HOXA5 promoter in the same strains. Extension of the 2 groups revealed a constant low HOXA5 methylation pattern in the controls in contrast to a
\end{abstract}

highly variable methylation pattern in the AIS patients. HOXA5 represents a candidate gene of androgen-mediated promoter methylation. The constantly low HOXA5 DNA methylation level of normal male scrotal fibroblast strains and the frequently high methylation levels in labia majora fibroblast strains in AIS indicate for the first time that androgen programming in sexual differentiation is not restricted to global gene transcription but also occurs at the epigenetic level.

Copyright $\odot 2011$ S. Karger AG, Base

Normal male sexual differentiation is dependent on the production of testosterone in the testes, its activation to dihydrotestosterone in the genital target tissues, and on the expression of a normal androgen receptor (AR). The latter is a ligand-activated transcriptional regulator belonging to the steroid receptor superfamily [Brinkmann et al., 1991]. Inactivating mutations of the X-chromosomal $A R$ gene lead to the androgen insensitivity syndrome (AIS) which is therefore a naturally occurring 'human disease model' for the role of androgen in human sexual development. AIS is clinically characterized by impaired genital virilization in $46, \mathrm{XY}$ individuals rang-

\section{KARGER}

Fax +4161306 1234

E-Mail karger@karger.ch

www.karger.com
(C) 2011 S. Karger AG, Basel

$1661-5425 / 11 / 0052-0070 \$ 38.00 / 0$

Accessible online at:

www.karger.com/sxd
Paul-Martin Holterhus

Department of Pediatrics, Christian Albrechts University (CAU) of Kiel and

University Hospital Schleswig-Holstein, Campus Kiel

Schwanenweg 20, DE-24105 Kiel (Germany)

Tel. +49 431597 1626, Fax +49 431597 3840,E-Mail holterhus@pediatrics.uni-kiel.de 
ing from complete AIS (CAIS) with phenotypically normal female external genitalia to varying degrees of partial AIS (PAIS; AIS4: predominantly female, AIS3: ambiguous, AIS2: predominantly male) with genital ambiguity to minimal AIS (MAIS) with only slightly diminished virilization or only infertility [Quigley et al., 1995].

Androgens are a special type of developmental hormone because they usually exert their biological effects via 2 timelines. On the one hand, androgens activate reversibly androgen receptor-mediated signaling in target cells [Werner et al., 2006]. On the other hand, there are certain sensitive windows during early embryogenesis and later post-natal life where androgens typically lead to irreversible anatomic changes thus resulting in the implementation of the normal male genital phenotype. Additional biological examples for irreversible androgen actions during male development are the pubertal changes of body shape including the male face [Schaefer et al., 2005] and growth of the larynx resulting in a deepening of the voice [Pedersen et al., 1986; Harries et al., 1998]. There is evidence for a sex-specific brain development and a particular role of prenatal programming of androgens therein [Döhler, 1985-1986; Hines, 2003; Jürgensen et al., 2007; Swaab, 2007].

More than 400 different $A R$ mutations associated with AIS have been identified to date (http://www.mcgill.ca/ androgendb/); however, there is not always a clear correlation between $A R$ mutation and clinical phenotype, mainly in PAIS [Evans et al., 1997; Boehmer et al., 2001b]. As possible causes of the phenotypic heterogeneity, AR somatic mosaicism, AR cofactors and differences in $5 \alpha$ reductase 2 activity were proposed in some cases [Holterhus et al., 1997, 1999; Adachi et al., 2000; Boehmer et al., 2001a]. A genome-wide approach unraveled the existence of sex-dimorphic gene expression patterns in cultured scrotal fibroblast strains of normal 46,XY individuals compared with 46,XY AIS patients [Holterhus et al., 2003, 2007]. While the gene expression patterns reflected differences in androgenization of the genitalia, the functional link between $A R$ mutation and altered gene expression patterns remained unclear.

In normal AR signaling, dihydrotestosterone is bound to the ligand binding domain (LBD) of the AR. This leads to a conformational change of the LBD which in turn leads to the release of molecular chaperones and to the recruitment of distinct coregulatory factors including enzymes that covalently modify histones and remodel chromatin [McEwan, 2004; Jia et al., 2006]. Comprehensive studies of the AR target gene KLK3 (kallikrein-related peptidase 3) revealed significant increase in histone H3-
K9/K14 acetylation and H3-K4 methylation in androgenindependent prostate cancer cells compared to their androgen-dependent counterparts [Jia et al., 2006]. Epigenetic changes at the DNA level therefore contribute to the control of transcription by androgens and the AR. This concept of hormonal control was also proven by a recent study that identified DNA methylation and demethylation by hormonal switching as main regulator for the function of the CYP27B1 gene promoter [Kim et al., 2009].

In an approach to identify epigenetic mechanisms that translate androgen receptor mutation into androgen-dependent gene expression patterns, we here compared the DNA methylation of labia majora fibroblast strains from 46,XY patients with AIS to scrotal fibroblast strains from normal 46,XY males.

\section{Materials and Methods}

The study has been approved by the ethical committee of the Christian Albrechts University of Kiel.

Fibroblast Strains, Cell Culture and Treatment Conditions

Scrotal-derived fibroblast strains were obtained from nine 46,XY males. Labia majora- or labioscrotal-derived fibroblast strains were obtained from fifteen $46, \mathrm{XY}$ patients with AIS and proven inactivating mutations of the $A R$ gene and two 46 , XY patients with AIS lacking a mutation in the $A R$ gene as previously described [Holterhus et al., 2003, 2007]. Fibroblasts were cultured to confluency on $150-\mathrm{mm}$ plastic dishes at $37^{\circ} \mathrm{C}$ with $5 \% \mathrm{CO}_{2}$. They were maintained in phenol-red-free DMEM F12 (Dulbecco's Modified Eagle Medium with the nutrient mix F12, Gibco/ Invitrogen, Karlsruhe, Germany) containing L-glutamine, $15 \mathrm{mM}$ Hepes buffer, penicillin/streptomycin (Gibco) and 12.9\% fetal calf serum (FCS, Gibco). Passage number of fibroblast culture and age at biopsy did not significantly differ in the 2 patient groups characterized by HOXA5 DNA promoter hypermethylation and HOXA5 DNA promoter hypomethylation.

\section{DNA Isolation}

DNA was isolated from fibroblasts using the DNeasy Blood \& Tissue protocol (Qiagen, Hilden, Germany).

DNA Methylation Profiling Using Universal Bead Arrays

Using the GoldenGate Methylation Cancer Panel I ${ }^{\circledR}$ (Illumina Inc., San Diego, Calif., USA) we have performed a pilot study to identify CpG loci differentially methylated between 2 AIS patients and 2 healthy controls. Both patients had CAIS due to inactivating $A R$ mutations. DNA methylation analyses were done as described previously [Martin-Subero et al., 2009a, b]. The array allows assaying 1,505 CpG sites from 807 selected genes, primarily focusing on genes relevant for tumorigenesis, differentiation, cell cycle control, and apoptosis. The reproducibility and accuracy of the GoldenGate Methylation Cancer Panel I based DNA methylation analysis has been demonstrated extensively [Bibikova et al., 2006; Martin-Subero et al., 2009b]. All analyses were 
performed in duplicate. CpG loci showing a delta beta value of at least 0.3 and an Illumina DiffScore above 30 or below -30 were considered as differentially methylated. The complete dataset is provided in online supplement table 1 (for all online suppl. material, see www.karger.com/doi/10.1159/000323807).

\section{Bisulfite Pyrosequencing}

Bisulfite pyrosequencing analysis of HOXA5 promoter methylation was performed according to standard protocols with slight modifications [Tost and Gut, 2007]. Genomic DNA was bisulfite converted using the EpiTect Bisulfite Conversion Kit (Qiagen). In a subsequent PCR amplification, locus-specific primers were used with one primer biotinylated at the $5^{\prime}$ end $\left(5^{\prime}\right.$-TTT TTG GTA AGT TTG GAA GTT AGG ATT TT-3'; 5'-AAA ACC CCA ACA AAA CCC AAT CT-3'). Amplification reactions contained approximately $75 \mathrm{ng}$ bisulfite-converted DNA, primers, AccuPrime Taq Polymerase, buffer II (Invitrogen), $50 \mathrm{mM} \mathrm{MgCl}_{2}$, and $2.5 \mathrm{mM}$ of each dNTP in a final volume of $50 \mu \mathrm{l}$. After initial denaturation, PCR consisted of 45 cycles of each $95^{\circ} \mathrm{C}$ for $30 \mathrm{~s}, 60^{\circ} \mathrm{C}$ for $30 \mathrm{~s}$, and $68^{\circ} \mathrm{C}$ for $30 \mathrm{~s}$, followed by a final synthesis at $68^{\circ} \mathrm{C}$ for $2 \mathrm{~min}$. PCR products were verified by gel electrophoresis. Single strands were prepared using the Vacuum Prep Tool (Biotage, Uppsala, Sweden) followed by a denaturation step at $85^{\circ} \mathrm{C}$ for $2 \mathrm{~min}$ and final sequencing primer ( $5^{\prime}$-GGT TTT GAA AGT TGT G-3') hybridisation. Pyrosequencing was performed using the Pyrosequencer ID and the DNA methylation analysis software Pyro Q-CpG 1.0.9 (Biotage), which was also used to quantify the ratio $\mathrm{T}: \mathrm{C}(\mathrm{mC}: \mathrm{C})$ at the analyzed $\mathrm{CpG}$ sites. Assays were validated using a commercial in vitro methylated DNA as positive control and whole genome amplification DNA as negative control (GE Healthcare, Munich, Germany). Principle component analysis has been performed using the Omics Explorer, Version 2.0 Beta (Qlucore AB, Lund, Sweden).

\section{RNA Preparation}

Total RNA was isolated from fibroblasts using Trizol (Invitrogen, Paisley, UK) followed by RNeasy mini kit protocol (Qiagen) according to the manufacturers' protocols. DNA contamination was removed using the DNA-free kit (Ambion, Austin, Tex., USA). Total RNA was quantified using the 2100 Bioanalyzer (Agilent, Palo Alto, Calif., USA).

\section{$q R T-P C R$}

Total RNA was amplified according to the manufacturer's instructions using the First Strand cDNA Synthesis kit (Fermentas, Hannover, Germany). qRT-PCR was performed using the IQ ${ }^{\mathrm{TM}}$ SYBR Green Supermix PCR kit (Bio-Rad Laboratories, Munich, Germany). The primers used for PCR (biomers.net GmbH, Ulm, Germany) had the following sequences: $H O X A 5$ forward primer 5'-GCA CAT AAG TCA TGA CAA CAT AG-3', and HOXA5 reverse primer 5'-CAG TAC TTT AAA CGC TCA GAT AC-3'. Hydroxymethylbilane synthase (HMBS) was used as housekeeping gene for normalization. $H M B S$ forward primer was 5 '-CTT CAC CAT CGG AGC CAT CTG C-3' and HMBS reverse primer was 5'-CGA AGC CGG GTG TTG AGG TTT-3'. PCR was carried out with the iCycler real time PCR detection system (Bio-Rad). We performed 2 independent experiments, each carried out as duplets resulting in 4 HOXA5 Ct values and 4 HMBS Ct values for each individual. For statistic analysis the mean $\mathrm{Ct}$ value for HOXA5 and HMBS was used for each individual.

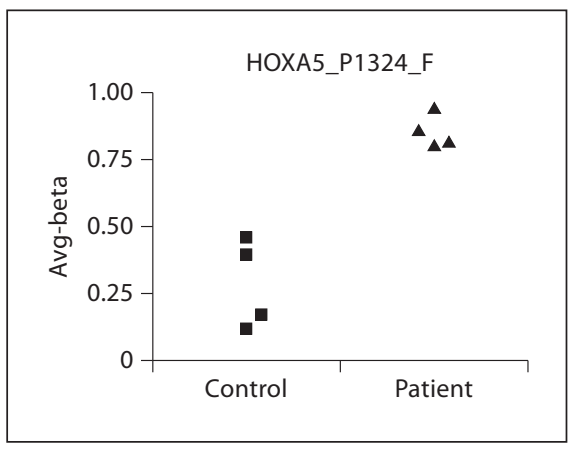

Fig. 1. Results from the GoldenGate Methylation Cancer Panel $1^{\circledR}$ methylation array. HOXA5_P1324_F indicates position chr7: 27,151,136 bp (NCBI Build 36.1). Avg-beta: methylation value. Patient: labia majora fibroblast strains from two 46,XY patients with CAIS due to $A R$ mutations. Control: 2 scrotal fibroblast strains from 46,XY male controls. All analyses were performed in duplicate.

For a $50-\mu$ l PCR master mix $25 \mu l$ of IQ ${ }^{\text {TM }}$ SYBR Green Supermix, $2 \mu \mathrm{l}$ of forward and reverse primer, respectively, $1 \mu \mathrm{l}$ of cDNA and water were mixed. After an activation step of $95^{\circ} \mathrm{C}$ for $7 \mathrm{~min}, 40$ amplification and detection cycles were performed $\left(95^{\circ} \mathrm{C}\right.$ for $30 \mathrm{~s}, 60^{\circ} \mathrm{C}$ for $45 \mathrm{~s}$ and $70^{\circ} \mathrm{C}$ for $\left.1 \mathrm{~min}\right)$. Melting curves were analyzed with the iCycler iQ optical System Software Version 3.0a (Bio-Rad). Fold changes were calculated using the $\Delta \mathrm{Ct}$ method [Bookout et al., 2006].

\section{Results}

\section{Array-Based Methylation Analysis}

Array-based DNA methylation analysis of 1,505 CpGs representing 807 genes using the Illumina GoldenGate Methylation Cancer Panel I demonstrated low DNA methylation values of HOXA5 at positions chr7: 27,149,625 bp and chr7: 27,151,136 bp (NCBI Build 36.1; Illumina HOXA5_E187_F and HOXA5_P1324_F) in scrotal fibroblast strains from two 46,XY normal males. In contrast, the same CpGs were hypermethylated in 2 labia majora fibroblast strains from 46,XY individuals with CAIS due to $A R$ mutations. A third CpG locus of the HOXA5 gene (NCBI Build 36.1; HOXA5_P479_F at position chr7: 27,150,291 bp) was found being equally methylated in both patient and control strains (see fig. 1 for HOXA5_P1324_F results).

\section{Bisulfite Pyrosequencing of the HOXA5 Promoter Region}

Quantitative methylation analysis of $13 \mathrm{CpG}$ positions in the promoter region of HOXA5 surrounding the $\mathrm{CpG}$ 
Fig. 2. Top: Unsupervised hierarchical cluster analysis of the methylation studies from $14 \mathrm{CpG}$ positions within the HOXA5 promoter region of labia majora fibroblast strains from fifteen $46, \mathrm{XY}$ patients with AIS and documented $A R$ mutation and scrotal fibroblast strains from nine 46,XY male controls. Included is the information of $\mathrm{AR} / A R$ mutation and genital phenotype for each individual tested. $A R$ mutations are signed according to the androgen receptor gene mutation database world-wide web server (http://androgendb.mcgill.ca/). CAIS: complete androgen insensitivity syndrome, female external genitalia; AIS4: slight virilization, predominantly female external genitalia; AIS3: ambiguous external genitalia; AIS2: predominantly male external genitalia; MAIS: male external genitalia. Red: high methylation level; green: low methylation level. Red squares indicate patients with various AIS degrees and documented $A R$ mutations. The green square indicates Scrotum 8 , an individual with an $A R$ mutation (c.2884T $>$ G, p.I841S) but normal male external genitalia (MAIS). Blue squares indicate normal male controls. Bottom: Results of a principle component analysis (PCA). PCA has been performed using the Omics Exporer, Version 2.0 Beta. PCA significantly separates a patient subgroup with HOXA5 DNA promoter hypermethylation from all male controls and a second subgroup of patients.

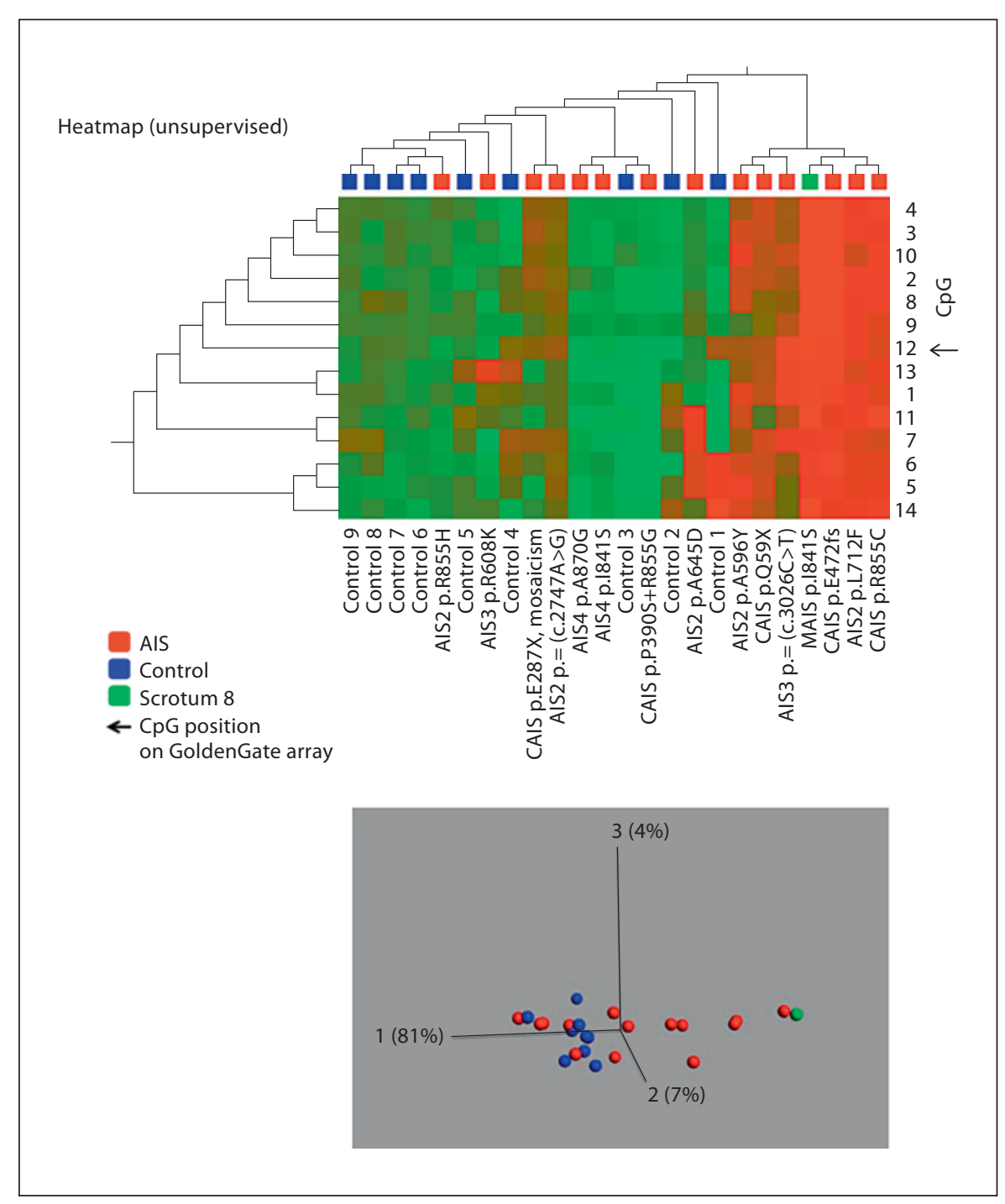

at position chr7: 27,151,136 bp which was previously investigated on the methylation array with bisulfite pyrosequencing revealed a mean DNA methylation range from 16 to $33 \%$ in 9 male 46 ,XY scrotal fibroblast strains (overall mean methylation 28\%). There was little variation reflected by a standard deviation of 5\%. The methylation analysis of 5 labia majora (CAIS), 9 labioscrotal (PAIS) and 1 scrotal (MAIS) fibroblast strains from 46,XY patients with $A R$ mutations and various degrees of AIS (according to table 1 in Holterhus et al. [2007]) revealed a striking variability of methylation patterns ranging from 14 up to $82 \%$ mean DNA methylation per case (overall mean methylation $41 \%$ ) with a standard deviation of $20 \%$ (online suppl. fig. 1). Thus, constantly low HOXA5 DNA methylation levels of normal male 46,XY scrotal fibro- blast strains are switched to high variability of HOXA5 DNA methylation levels in labioscrotal fibroblast strains of patients with $A R$ mutations and various degrees of AIS phenotype.

Unsupervised hierarchical cluster analysis of the HOXA5 DNA methylation values derived from bisulfite pyrosequencing analyses identified 2 different groups within all tested individuals. Remarkably, all 9 male controls and 8 of 15 AIS patients (2 CAIS, 2 AIS4, 1 AIS3, and 3 AIS2 patients) showed low HOXA5 DNA methylation. In contrast, 7 from 15 AIS patients (3 CAIS, 1 AIS3, 2 AIS2 and 1 MAIS patient [Scrotum 8; Appari et al., 2009]) but none of the normal male controls showed HOXA5 DNA hypermethylation (fig. 2). The patients with $A R$ mutations significantly more frequently showed high 
methylation values than normal controls ( $\mathrm{p}=0.02$, Fisher's exact test).

Out of the group with low HOXA5 DNA methylation, there is 1 CAIS patient, who is known to be mosaic for an AR mutation (ARD 465, p.E287X [Holterhus et al., 2003]).

One individual with normal male phenotype, who initially presented with infertility, carried an androgen receptor mutation (c.2884T $>$ G, p.I841S [Appari et al., 2009]). Remarkably, despite the normal male genital phenotype he showed an extremely high HOXA5 promoter methylation pattern with a mean methylation value of $82 \%$. The identical $A R$ mutation (c.2884T $>$ G, p.I841S) was also observed in an AIS4 patient who showed a mean HOXA5 promoter methylation of only $20 \%$. These results indicate that there is no direct correlation between a given $A R$ mutation and HOXA5 DNA methylation levels in the analyzed fibroblast strains of the corresponding individuals.

Moreover, 2 additional patients with AIS (CAIS, AIS3) who lacked an androgen receptor mutation by sequencing [Holterhus et al., 2005] showed very low HOXA5 promoter methylation patterns with mean methylation values of 11 and 14\%, similar to the HOXA5 DNA methylation levels of the 9 male controls (data not shown). Thus, no obvious association of the HOXA5 DNA methylation level in the analyzed fibroblast strains and the AIS phenotype was detectable.

\section{qRT-PCR of HOXA5}

We studied expression of $H O X A 5$ transcripts in 8 male control scrotal fibroblast strains and 14 AIS labioscrotal fibroblast strains by qRT-PCR. HOXA5 methylation of the $\mathrm{CpG}$ loci under study did not lead to HOXA5 gene repression. Instead, we observed a significant $(\mathrm{p}=0.01)$ linear relationship between increasing HOXA5 DNA methylation values and increasing HOXA5 expression with an $r$ of 0.52 and an $r^{2}$ of 0.271 .

\section{Discussion}

Here we describe HOXA5 as significantly hypermethylated in 2 CAIS patients compared to 2 normal male controls in an explorative screening of global genital fibroblast methylation status using the GoldenGate Methylation Cancer Panel I microarray. Given the involvement of HOXA5 as member of a conserved family of transcription factors in embryogenesis, tissue-specific development and cell differentiation [Scott et al., 2005], its interplay with hormones and its influence in development of reproductive tissues through its downstream target genes IGFBP1, PTN and the progesterone receptor PGR [Svingen and Tonissen, 2006], HOXA5 seemed to be a promising candidate gene for further investigation of DNA methylation in AIS. Therefore, we performed an independent validation of our methylation array experiments in fifteen 46,XY labia majora and labioscrotal fibroblast strains from patients with documented $A R$ mutations and various degrees of AIS, as well as in 9 scrotal fibroblast strains from 46,XY male controls. Hershko et al. [2002] previously reported that methylation of HOXA5 occurred only in $\mathrm{CpG}$ island sequences. Thus, our bisulfite pyrosequencing study design was set up accordingly by analyzing $14 \mathrm{CpG}$ positions in the $\mathrm{CpG}$ island of the HOXA5 promoter region.

We could reproduce the results for the 4 individuals on the methylation array by bisulfite pyrosequencing, confirming a good correlation between the 2 independent methods used. Moreover, we observed that HOXA5 promoter methylation patterns of all additionally tested male controls remained stably low, while the HOXA5 promoter methylation patterns of the AIS patients revealed a striking variability with 7 of 15 AIS patients showing HOXA5 hypermethylation and 8 of 15 patients showing low HOXA5 methylation levels. These observations could support the hypothesis that HOXA5 promoter methylation is controlled by the androgen receptor and that a constantly low HOXA5 methylation pattern is usually associated with, but not sufficient for, normal male genital development, as can be observed in some CAIS patients having as low HOXA5 methylation levels as normal male controls (fig. 2). On the one hand, this indicates that the AR must have additional influences on other genes beside HOXA5 and is thereby probably contributing on multiple redundant sites in a whole gene network to the virilization deficit in AIS patients. On the other hand, the observed differences of HOXA5 DNA methylation levels even in the case of identical $A R$ mutations (e.g. c.2884T $>$ G, p.I841S) indicate that HOXA5 DNA methylation control is not restricted to the $A R$ gene but rather involves different other genes, probably at different time windows and to a different extent. Thus, our experimental data suggest that androgen-regulated genital differentiation is embedded in a coordinated developmental network involving the androgen receptor as an important, but not exclusive, factor. The phenomenon that transcriptional control of a gene is not restricted to the influence of a single gene is well known due to the fact that e.g. AR-binding sequences (ARE, ARR or HRE) occur next to binding sites for housekeeping (Sp1 [Chen 
et al., 1997], CCAAT and NF-1 [Song et al., 1999]), inducible (NF-кB [Supakar et al., 1995], Ets and AP-1 [McEwan, 2004]) and tissue-specific transcription factors [McEwan, 2004]. However, how the implementations of epigenetic changes occur in the case of HOXA5 and which additional genes beside $A R$ are involved may be entirely unrelated to our current knowledge of androgen-related transcriptional control. Estrogens do not play a leading role in sex-specific morphogenesis of the external genitalia. However, AIS is generally characterized by an increased conversion of testosterone to estradiol by aromatization. Therefore, we cannot exclude that part of the HOXA5 promoter methylation patterns may have been modulated via the estrogen receptor pathway in the AIS samples, thus contributing to the observed epigenetic variability. In the same way, prenatal influences through medication or pregnancy course may affect methylation of the HOXA5 promoter and are of high interest for further studies in the field of AIS phenotypic heterogeneity.
In conclusion, we show that HOXA5 represents a candidate gene of androgen-mediated promoter methylation. We thereby suggest the concept that epigenetic changes may function as long-lasting androgen memory, supporting our earlier genome-wide gene expression studies in fibroblasts and in blood [Holterhus et al., 2003, 2007, 2009]. While we were neither able to link the HOXA5 DNA methylation levels to a distinct AIS phenotype nor to a distinct $A R$ mutation, the coordinated interplay of genes involved in the complex network that contributes to male genital differentiation could possibly explain PAIS heterogeneity (online suppl. fig. 2).

\section{Acknowledgements}

The authors thank Kerstin Runde and Tanja Dahm for their excellent technical assistance. The research leading to these results has received funding from the European Community's Seventh Framework Program (FP7/2007-2013) under grant agreement No. 201444 (WP4 to R.S. and P.-M.H.).

\section{References}

Adachi M, Takayanagi R, Tamori A, Imasaki K, Kato $S$, et al: Androgen-insensitivity syndrome as a possible coactivator disease. $\mathrm{N}$ Engl J Med 343:856-862 (2000).

-Appari M, Werner R, Wünsch L, Cario G, Demeter J, et al: Apolipoprotein D (APOD) is a putative biomarker of androgen receptor function in androgen insensitivity syndrome. J Mol Med 87:623-632 (2009).

-Bibikova M, Lin Z, Zhou L, Chudin E, Garcia EW, et al: High-throughput DNA methylation profiling using universal bead arrays. Genome Res 16:383-393 (2006).

- Boehmer ALM, Brinkmann AO, Nijman RM, Verleun-Mooijman MC, de Ruiter P, et al: Phenotypic variation in a family with partial androgen insensitivity syndrome explained by differences in 5 alpha dihydrotestosterone availability. J Clin Endocrinol Metab 86: 1240-1246 (2001a).

-Boehmer ALM, Brüggenwirth H, van Assendelft C, Otten BJ, Verleun-Mooijman MC, et al: Genotype versus phenotype in families with androgen insensitivity syndrome. J Clin Endocrinol Metab 86:4151-4160 (2001b).

Bookout AL, Cummins CL, Mangelsdorf DJ, Pesola JM, Kramer MF: High-throughput realtime quantitative reverse transcription PCR. Curr Protoc Mol Biol 15:Unit 15.18 (2006).

-Brinkmann AO, Kuiper GG, Ris-Stalpers C, van Rooij HC, Romalo G, et al: Androgen receptor abnormalities. J Steroid Biochem Mol Biol 40:349-352 (1991).
Chen S, Supakar PC, Vellanoweth RL, Song CS, Chatterjee B, Roy AK: Functional role of a conformationally flexible homopurine/homopyrimidine domain of the androgen receptor gene promoter interacting with $\mathrm{Sp} 1$ and a pyrimidine single strand DNA-binding protein. Mol Endocrinol 11:3-15 (1997).

Döhler KD: Development and differentiation of the sexually dimorphic nucleus in the preoptic area of the rat brain is under hormonal control. Int J Neurol 19-20:218-222 (19851986).

-Evans BAJ, Hughes IA, Bevan CL, Patterson MN, Gregory JW: Phenotypic diversity in siblings with partial androgen insensitivity syndrome. Arch Dis Child 76:529-531 (1997).

-Harries M, Hawkins S, Hacking J, Hughes IA: Changes in the male voice at puberty: vocal fold length and its relationship to the fundamental frequency of the voice. J Laryngol Otol 112:451-454 (1998).

Hershko A, Kafri T, Fainsod A, Razin A: Methylation of HoxA5 and HoxB5 and its relevance to expression during mouse development. Gene 302:65-72 (2002)

Hines M: Sex steroids and human behavior: Prenatal androgen exposure and sex-typical play behavior in children. Ann N Y Acad Sci 1007:272-282 (2003).
Holterhus PM, Brüggenwirth HT, Hiort O, Kleinkauf-Houcken A, Kruse K, et al: Mosaicism due to a somatic mutation of the androgen receptor gene determines phenotype in androgen insensitivity syndrome. J Clin Endocrinol Metab 82:3584-3589 (1997).

-Holterhus PM, Wiebel J, Sinnecker GH, Brüggenwirth HT, Sippell WG, et al: Clinical and molecular spectrum of somatic mosaicism in androgen insensitivity syndrome. Pediatr Res 46:684-690 (1999).

- Holterhus PM, Hiort O, Demeter J, Brown PO, Brooks JD: Differential gene-expression patterns in genital fibroblasts of normal males and 46,XY females with androgen insensitivity syndrome: evidence for early programming involving the androgen receptor. Genome Biol 4:R37 (2003).

-Holterhus PM, Werner R, Hoppe U, Bassler J, Korsch E, et al: Molecular features and clinical phenotypes in androgen insensitivity syndrome in the absence and presence of androgen receptor gene mutations. J Mol Med 83:1005-1013 (2005).

Holterhus PM, Deppe U, Werner R, Richter-Unruh A, Bebermeier J-H, et al: Intrinsic androgen-dependent gene expression patterns revealed by comparison of genital fibroblasts from normal males and individuals with complete and partial androgen insensitivity syndrome. BMC Genomics 8:376 (2007). 
-Holterhus PM, Bebermeier JH, Werner R, Demeter J, Richter-Unruh A, et al: Disorders of sex development expose transcriptional autonomy of genetic sex and androgen-programmed hormonal sex in human blood leukocytes. BMC Genomics 10:292 (2009).

- Jia L, Shen HC, Wantroba M, Khalid O, Liang G, et al: Locus-wide chromatin remodeling and enhanced androgen receptor-mediated transcription in recurrent prostate cancer cells. Mol Cell Biol 26:7331-7341 (2006).

Jürgensen M, Hiort O, Holterhus PM, Thyen U: Gender role behavior in children with $\mathrm{XY}$ karyotype and disorders of sex development. Horm Behav 51:443-453 (2007).

-Kim M-S, Kondo T, Takada I, Youn M-Y, Yamamoto $Y$, et al: DNA demethylation in hormone-induced transcriptional derepression. Nature 461:1007-1012 (2009).

-Martin-Subero JI, Ammerpohl O, Bibikova M, Wickham-Garcia E, Agirre X, et al: A comprehensive microarray-based DNA methylation study of 367 hematological neoplasms. PLoS One 4:e6986 (2009a).
Martin-Subero JI, Kreuz M, Bentink S, Ammerpohl O, Wickham-Garcia E, et al: New insights into biology and origin of mature aggressive B-cell lymphomas by combined epigenomic, genomic, and transcriptional profiling. Blood 113:2488-2497 (2009b).

McEwan IJ: Molecular mechanisms of androgen receptor-mediated gene regulation: structure-function analysis of the AF-1 domain. Endocr Relat Cancer 11:281-293 (2004).

-Pedersen MF, Moller S, Krabbe S, Bennett P: Fundamental voice frequency measured by electroglottography during continuous speech. A new exact secondary sex characteristic in boy in puberty. Int J Pediatr Otorhinolaryngol 11:21-27 (1986).

Quigley CA, De Bellis A, Marschke KB, elAwady MK, Wilson EM, French FS: Androgen receptor defects: Historical, clinical, and molecular perspectives. Endocr Rev 16:271321 (1995).

Schaefer K, Fink B, Mitteroecker P, Neave N, Bookout FL: Visualizing facial shape regression upon 2 nd to 4 th digit ratio and testosterone. Coll Antropol 29:415-419 (2005).

- Scott V, Morgan EA, Stadler HS: Genitourinary functions of Hoxa13 and Hoxd13. J Biochem 137:671-676 (2005).
-Song CS, Jung MH, Supakar PC, Chatterjee B, Roy AK: Negative regulation of the androgen receptor gene promoter by NF1 and adjacently located multiprotein-binding site. Mol Endocrinol 13:1487-1496 (1999).

- Supakar PC, Jung MH, Song CS, Chatterjee B, Roy AK: Nuclear factor kappa B functions as a negative regulator for the rat androgen receptor gene and NF-kappa B activity increases during the age-dependent desensitization of the liver. J Biol Chem 270:837-842 (1995).

Svingen T, Tonissen KF: Hox transcription factors and their elusive mammalian gene targets. Heredity 97:88-96 (2006).

Swaab DF: Sexual differentiation of the brain and behavior. Best Pract Res Clin Endocrinol Metab 21:431-444 (2007).

- Tost J, Gut IG: DNA methylation analysis by pyrosequencing. Nat Protoc 2:2265-2275 (2007).

-Werner R, Schütt J, Hannema S, Röpke A, Wieacker P, et al: Androgen receptor gene mutations in androgen insensitivity syndrome cause distinct patterns of reduced activation of androgen-responsive promoter constructs. J Steroid Biochem Mol Biol 101: 1-10 (2006). 